Europhys. Lett., 65 (1), pp. 130-136 (2004)

DOI: $10.1209 / \mathrm{epl} / \mathrm{i} 2003-10054-\mathrm{x}$

\title{
Muon spin relaxation as a probe of potential carbon-based hydrogen absorbers
}

\author{
T. P. Blach and E. MacA. Gray $\left(^{*}\right)$ \\ Physics Group, School of Science, Griffith University - Brisbane 4111, Australia
}

(received 7 August 2003; accepted in final form 28 October 2003)

PACS. 76.75.+i - Muon spin rotation and relaxation.

PACS. 81.05. Uw - Carbon, diamond, graphite.

PACS. 82.75.-z - Molecular sieves, zeolites, clathrates, and other complex solids.

\begin{abstract}
Literature reports of hydrogen absorption by nanostructured carbons range from nothing to tens of weight percent. We studied pristine graphite and two potassium-graphite intercalation compounds (GICs) using muon spin relaxation $(\mu \mathrm{SR})$, because the positive muon is expected to behave like a light hydrogen isotope. The graphite did not trap muons, but at low temperatures a component of the $\mu \mathrm{SR}$ spectrum from the two GICs could only be modelled by the Kubo-Toyabe function. This result demonstrates that muons were trapped by these samples and implies that $\mathrm{H}$ atoms would also be localised in these traps, in qualitative agreement with accepted hydrogen absorption results. Thus $\mu \mathrm{SR}$ is confirmed to be a valuable probe of the relative ability of carbon-based samples to bind hydrogen atoms.
\end{abstract}

Introduction. - The basic problem of storing hydrogen in solid form at near-ambient temperature and pressure is the mass of absorbed hydrogen relative to the mass of the host. Despite some 30 years of effort, the best commercially available metallic storage hosts absorb only about $3 \mathrm{wt} \%$ of atomic hydrogen, in contrast to the $17 \mathrm{wt} \%$ hydrogen in automotive petroleum.

The hydrogen storage capacity of metals extends to a hydrogen-per-metal atomic ratio $(\mathrm{H} / \mathrm{M})$ between one and two in the best examples. The concept of replacing the heavy metallic host matrix with a carbon-based material has obvious attraction because of the relatively low atomic mass of carbon: achieving a recoverable hydrogen-per-carbon ratio $\mathrm{H} / \mathrm{C}=1$ in a pure carbon host would correspond to a technologically exciting $7.7 \mathrm{wt} \%$ hydrogen.

The absorption of hydrogen by carbon-based materials is a current major controversy in materials science. Carbon-based materials such as nanotubes, nanofibres and GICs have been reported to achieve very high hydrogen storage capacities, some even exceeding $50 \mathrm{wt} \%$ [1-4]. If even the more modest claims are true, carbon-based materials promise a new era in hydrogen storage. There is however no consensus on hydrogen absorption by carbons beyond about one weight percent, and a recipe for high storage capacity reported by one group is typically unsuccessful when followed by another group. Some of the published claims have been recently reviewed by Schlapbach and Züttel [5], Hirscher et al. [6] and Gundiah et al. [7]. On the

$\left({ }^{*}\right)$ E-mail: E.Gray@griffith.edu.au

(C) EDP Sciences 
assumption that not all researchers who claim capacities greater than $1 \mathrm{wt} \%$ are likely to be wrong, the problem of hydrogen storage in carbon-based materials is evidently a complex one, possibly controlled by as yet unknown factors of materials preparation or composition.

Our aim in the work reported here was to step outside the controversy over preparation methods and measurement techniques for determining hydrogen uptake, and to focus on the basic structural properties of carbons in relation to hydrogen. We thereby hoped to gain some insight into whether dissociated hydrogen atoms (as distinct from $\mathrm{H}_{2}$ molecules) should be expected to be absorbed or not to be absorbed by particular carbon-based materials.

We used muon spin relaxation $(\mu \mathrm{SR})$ because the positive muon has characteristics which are unique in the present context. First, it is expected to behave rather like a proton in a host structure and to be trapped in sites where $\mathrm{H}$ would be trapped. Being lighter than a proton (207 electron masses), the muon diffuses faster and may not enter a bound state when $\mathrm{H}$ would, by virtue of its higher zero-point energy. In this respect it may be considered a "pessimistic" probe, in that strong muon trapping should indicate even stronger hydrogen trapping. Additionally, muons can be injected into the sample with high initial momentum (about $28 \mathrm{MeV} / c$ in our experiment), so surface effects hindering the dissociation of molecular hydrogen or penetration by $\mathrm{H}$ to the interior of the sample can be circumvented by $\mu \mathrm{SR}$.

Potassium-intercalated graphite is among the candidates for which claims of high capacity have been made [4] and subsequently discredited [8,9] as artefacts of the experimental technique used to measure hydrogen uptake. A more modest claim, based on credible experiments, has also been made for one potassium-intercalated graphite, which was found to absorb hydrogen to the equivalent of $\mathrm{C}_{8} \mathrm{KH}_{4.5}$, or $3.4 \mathrm{wt} \%$, at $78 \mathrm{~K}$ under 2000 bar $\mathrm{H}_{2}$ [10].

Carbon has no nuclear magnetic moment and, in "normal" graphite, no localised electronic moment. Therefore, the muon-carbon interaction is weak, so intercalates in which the dopant atoms have a nuclear moment are more favourable subjects for $\mu \mathrm{SR}$ than pure graphite. Although pure graphite has been studied by $\mu \mathrm{SR}[11,12]$, GICs have not. Our aim was therefore to see what effect intercalated potassium had on the diffusion of muons and to relate the results to the accepted hydrogen absorption capacities of graphite (essentially zero) and the intercalates, which are generally acknowledged to absorb to at least $\mathrm{C}_{8} \mathrm{KH}_{2 / 3}$ [13] and $\mathrm{C}_{24} \mathrm{KH}_{4}$ [14].

Experimental details. - We used the pulsed muon beam facility at ISIS, Rutherford Appleton Laboratory, England. In the $\mu \mathrm{SR}$ technique pulses of $100 \%$ spin-polarised muons are injected into the sample. The process of thermalisation by multiple "collisions" with the atoms of the sample is very fast, so muons do not lose their polarisation unless very strong internal magnetic fields are present. Following thermalisation, the muon precesses about the local magnetic field. The muon decays with a lifetime of $2.2 \mu \mathrm{s}$, producing a positron preferentially emitted along the muon's spin axis at the instant of its decay. $\mu$ SR spectra take the form of asymmetry of positron counts vs. time. In favourable cases much information about muon diffusion and trapping can be obtained, including knowledge of the particular crystallographic site(s) occupied by the muon if a single crystal is available.

In the case of graphite and the GICs we studied, the only significant contribution to the local field comes from the potassium nuclear moment $\left(0.6 \mu_{\mathrm{N}}\right)$. Our $\mu \mathrm{SR}$ experiments were carried out on powdered samples. Zero-field measurements were made with the EMU spectrometer. The same sequence of temperatures ranging from $5 \mathrm{~K}$ to $350 \mathrm{~K}$ was followed for each sample. The spectra were analysed using the WIMDA modelling package [15], with which the profile of a spectrum can be tested against standard forms deriving from particular physical models of the structure and dynamics of the sample-muon system.

Three samples were studied: pristine graphite starting material (Ceylon natural graphite) 


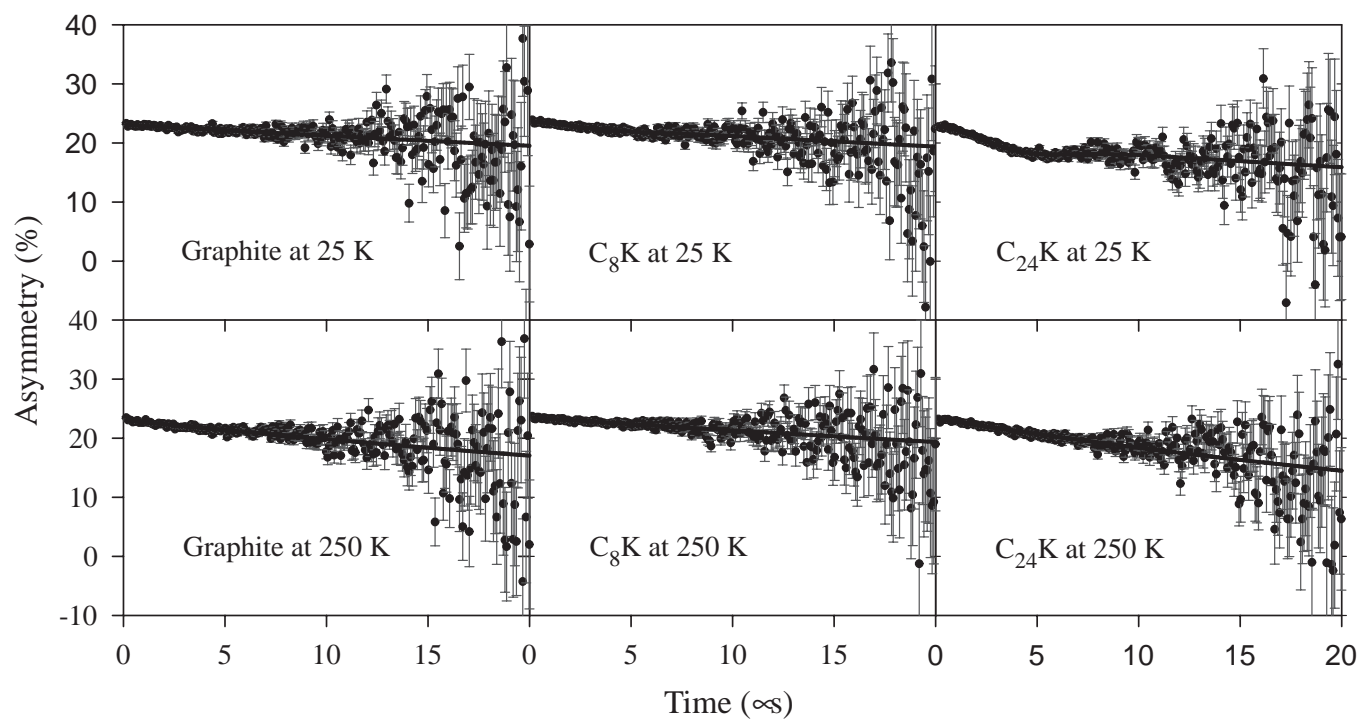

Fig. 1 - Zero-field muon relaxation spectra for graphite, $\mathrm{C}_{8} \mathrm{~K}$ and $\mathrm{C}_{24} \mathrm{~K} . \mathrm{C}_{24} \mathrm{~K}$ shows evidence of enhanced muon trapping at low temperatures, as indicated by the Kubo-Toyabe dip, which is present but much weaker in $\mathrm{C}_{8} \mathrm{~K}$.

and two GICs made from it, $\mathrm{C}_{24} \mathrm{~K}$ and $\mathrm{C}_{8} \mathrm{~K}$. The GICs were made by heating stoichiometric amounts of graphite and potassium to $300^{\circ} \mathrm{C}$ in an evacuated and sealed glass tube for several days and had the expected uniform deep blue and gold colours, respectively. Neutron diffraction patterns showed that they had good crystallinity. The samples were loaded into stainless-steel shipping cells under continuously purified argon, to minimise the effects of atmospheric water and oxygen, taken to ISIS and then transferred into titanium sample holders sealed with indium gaskets, again under continuously purified argon.

Results. - Figure 1 shows zero-field $\mu \mathrm{SR}$ spectra collected from pristine graphite, $\mathrm{C}_{8} \mathrm{~K}$ and $\mathrm{C}_{24} \mathrm{~K}$. The loss of asymmetry in graphite was minimal in the experimental time window (about $15 \mu \mathrm{s}$ in this case), owing to the weak muon-carbon interaction. At temperatures above $100 \mathrm{~K}$, the graphite spectra were not well described by a single decay term and were fitted by a sum of two exponential functions:

$$
G_{z}=A_{1} \exp \left[-\lambda_{1} t\right]+A_{2} \exp \left[-\lambda_{2} t\right]
$$

where $A_{1}$ and $A_{2}$ are the initial asymmetries of the damping functions and $\lambda_{1}$ and $\lambda_{2}$ are damping parameters typically measured in $\mathrm{kHz}$ and related to muon diffusion rates. Although the proportion of the second signal was small (up to $5 \%$ at room temperature), the relaxation of this component was much faster than in the majority signal and its rate was fixed to $460 \mathrm{kHz}$ throughout the fitting process. $A_{2}$ did not change within experimental error in the temperature range of $150 \mathrm{~K}-300 \mathrm{~K}$ and vanished altogether at temperatures below $100 \mathrm{~K}$. This behaviour was not observed in the intercalates and was not previously reported by other researchers studying graphite [11].

The spectra from the two intercalates reveal a "Kubo-Toyabe dip". Unlike the exponential function used to model rapidly diffusing muons, the Kubo-Toyabe (KT) function applies to muons that are either trapped or diffuse on a time scale longer than the experimental window. 


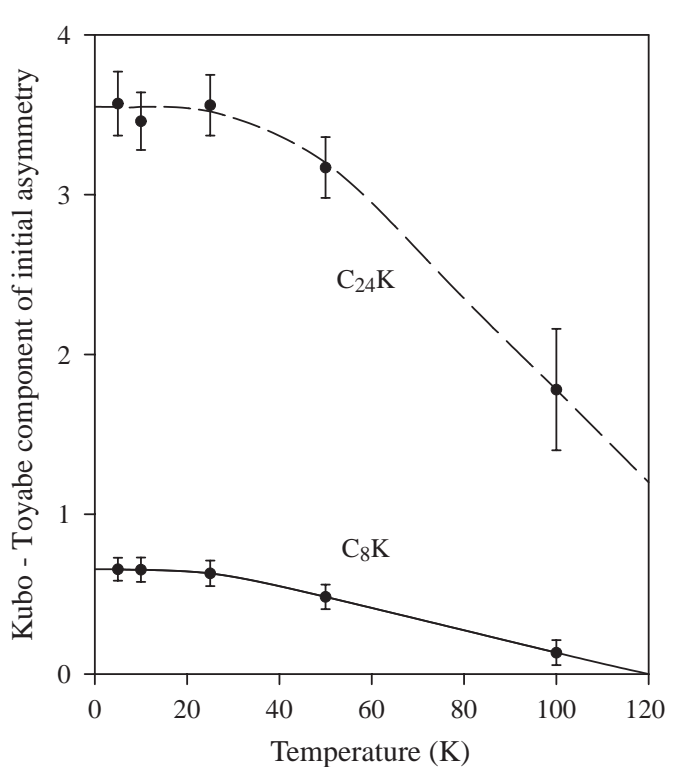

Fig. 2

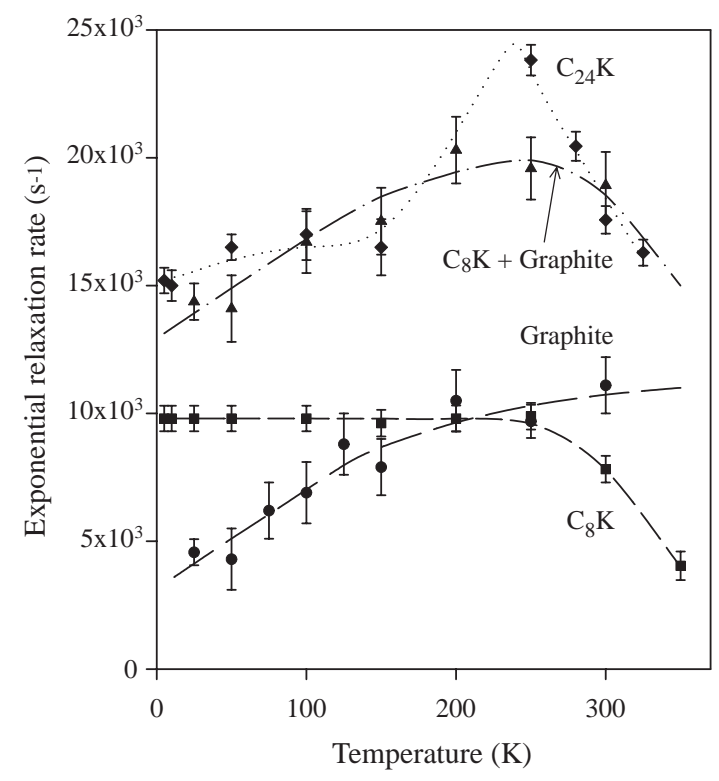

Fig. 3

Fig. 2 - Initial asymmetry of the Kubo-Toyabe component of the fitting function (eq. (2)), demonstrating the increased likelihood of muon trapping at low temperatures. The sum of this component and the initial asymmetry of the exponential was constant at $24 \%$ and independent of temperature. Pure graphite showed no evidence of Kubo-Toyabe behaviour.

Fig. 3 - Relaxation rate of the exponential component of the fitting function, applying to rapidly diffusing muons. The relaxation rate for graphite is somewhat higher than reported by Cox et al. [12], but follows the same trend. $\mathrm{C}_{24} \mathrm{~K}$ resembles a mixture of the graphite and $\mathrm{C}_{8} \mathrm{~K}$ structures.

The appearance of a KT dip is thus synonymous with muon trapping by the host [16]. The KT function alone fitted these spectra poorly at short times, where the statistics are best, so they were fitted with the sum of an exponential function and a KT term:

$$
G_{z}=A_{1} \exp \left[-\lambda_{1} t\right]+A_{2}\left\{\frac{1}{3}+\frac{2}{3}\left(1-(\Delta t)^{2}\right) \exp \left[-\frac{(\Delta t)^{2}}{2}\right]\right\},
$$

where the parameter $\Delta$ is related to the width of the local field distribution. A large value of $\Delta$ reflects a strong dipolar interaction between the muon and its host. $\Delta$ was found to have the same value for the two intercalates, $330 \pm 20 \mathrm{kHz}$, equivalent to a width of the local field distribution at the muon trap sites of $4.0 \pm 0.2$ gauss.

The initial asymmetry $A_{2}$, extracted by fitting eq. (2) to the $\mathrm{C}_{24} \mathrm{~K}$ and $\mathrm{C}_{8} \mathrm{~K}$ spectra, depends on the number of muons that are trapped (fig. 2). The sum of initial asymmetries $A_{1}+A_{2}$ remained constant throughout the temperature range of the measurements, confirming that the fitting functions and procedure are reasonable and that there were no losses in muon injection. The proportion of trapped muons in $\mathrm{C}_{24} \mathrm{~K}$ is evidently much higher than in $\mathrm{C}_{8} \mathrm{~K}$.

The behaviour of the damping parameter $\lambda_{1}$ (fig. 3) shows that the three materials are very different as viewed by the muon. Although the relaxation rates are small, the rate increases with temperature in pure graphite, decreases with temperature in $\mathrm{C}_{8} \mathrm{~K}$ and experiences a local peak at about $230 \mathrm{~K}$ in $\mathrm{C}_{24} \mathrm{~K}$. The relaxation rate for $\mathrm{C}_{24} \mathrm{~K}$ roughly follows the sum of 
the graphite and $\mathrm{C}_{8} \mathrm{~K}$ relaxation rates, suggesting that muons view $\mathrm{C}_{24} \mathrm{~K}$ as a mixture of the other two materials.

Discussion. - The significance of our results hinges on the correlation between muon trapping and the imputed potential to trap hydrogen atoms, as distinct from molecules. The theoretical basis for this correlation has not been generally established to our knowledge. In this context, we point out that graphite and GICs are better viewed as near-metals owing to the gapless band structure of graphite. Furthermore, there is no experimental evidence of muonium formation (observed in insulators) in our results or in the previous studies of graphite $[11,12]$. A connection between muon trapping and hydrogen trapping in metals is explicitly supported by first-principles calculations [17] for positive interstitial impurities in palladium, where the increasing zero-point energy of the progressively lighter $\mathrm{T}, \mathrm{D}, \mathrm{H}$ and $\mu^{+}$hydrogen isotopes bound to interstitial holes was demonstrated. A qualitatively similar relationship between the vibration energies of the various hydrogen isotopes in graphite therefore seems plausible.

Our results confirm that crystalline graphite does not trap muons appreciably, consistent with the accepted experimental fact that regular (not nanostructured by ball milling, for example) graphite does not absorb more than a tiny quantity of hydrogen. The absence of muon trapping is not sufficient to disprove hydrogen trapping, however, as a muon-host interaction via magnetic fields is required to observe the trapping. This point is further considered below.

In contrast, where muon trapping is observable via a strong muon-host interaction, the muon-hydrogen analogy points to the trapping of hydrogen atoms. The increase in the initial asymmetry of the Kubo-Toyabe spectral component in going from $\mathrm{C}_{8} \mathrm{~K}$ to $\mathrm{C}_{24} \mathrm{~K}$, despite the lower concentration of $\mathrm{K}$ atoms, correlates nicely with the accepted higher hydrogen capacity of $\mathrm{C}_{24} \mathrm{~K}$. Our results for $\mathrm{C}_{8} \mathrm{~K}$ suggest that much more hydrogen should be trapped as $\mathrm{H}$ at low temperatures than at room temperature, in qualitative agreement with the finding [10] that $\mathrm{C}_{8} \mathrm{~K}$ absorbs around five times as much hydrogen at $78 \mathrm{~K}$ compared to the same pressure at room temperature.

Since natural carbon has practically no nuclear magnetic moment, the precession measured by $\mu \mathrm{SR}$ must come from either proximity to the weak nuclear moment of the $\mathrm{K}$ atoms or from modification of the local electronic environment owing to the insertion of $\mathrm{K}$. Whichever is the source of the muon-host interaction, the fact that the value of $\Delta$ is the same in $\mathrm{C}_{24} \mathrm{~K}$ and $\mathrm{C}_{8} \mathrm{~K}$ suggests that the muon is trapped at the same site in both structures, with the same proximity to the $\mathrm{K}$ atom. The question is then why the probability of trapping is so much greater in the GIC with less intercalated K, as shown by fig. 2 .

The initial asymmetry data in fig. 3 may offer a clue in that $\mathrm{C}_{24} \mathrm{~K}$ resembles a mixture of graphite and $\mathrm{C}_{8} \mathrm{~K}$ as far as the muon is concerned. The staging sequence in the two intercalates is ideally ...-C-K-C-K-C- . . in $\mathrm{C}_{8} \mathrm{~K}$ (a stage-1 GIC) and . . -C-C-K-C-C-K-C-C- . . in $\mathrm{C}_{24} \mathrm{~K}$ (a stage-2 GIC in which the areal density of $\mathrm{K}$ atoms is $2 / 3$ that in $\mathrm{C}_{8} \mathrm{~K}$ ). In terms of the interstices between graphite sheets, where we expect muons and $\mathrm{H}$ to be localised, $\mathrm{C}_{24} \mathrm{~K}$ is indeed a mixture of pure graphite $\mathrm{C}-\mathrm{C}$ layers and $\mathrm{C}_{8} \mathrm{~K}$ C-K-C layers. It has been proposed [18] that stage-2 GICs contain large regions of stage-1 stacking with diluted potassium planes, and this picture is at least qualitatively consistent with our results. This leads us to speculate that the muon trap sites may be point defects such as potassium vacancies, which could well be more numerous in the stage-2 GIC.

The translation of muon trapping probability into $\mathrm{H}$ absorption capacity is difficult, because muons decay in traps and thereby vacate them for further trapping or diffusion events. The total number of incident muons in an experiment is likely to be small compared to the 
number of trap sites, however, so the thermalisation and subsequent decay of two muons in the same trap site may be unlikely. In contrast, stable $\mathrm{H}$ atoms occupy traps permanently, even if they diffuse between traps. Thus, the muon may be regarded as highly sensitive to $\mathrm{H}$ trapping potential, but the corresponding real $\mathrm{H}$ capacity may be smaller than the muon trapping data suggest at first sight. This would certainly be the case if the trap sites were indeed $\mathrm{K}$ vacancies.

The condition of the graphite starting material has been reported to greatly affect the absorption of small quantities of hydrogen by GICs [18], and the occurrence of a second exponential term in the graphite $\mu \mathrm{SR}$ spectra, which was not previously observed [11], may indicate a relevant special property of our natural graphite samples.

Hydrogen dissociation is not a problem in absorption experiments with potassium intercalates, which are believed to catalyse $\mathrm{H}_{2}$ dissociation [19]. It is likely, however, that some potential hydrogen absorbers suffer from surface-related problems which prevent $\mathrm{H}_{2}$ dissociation, or pose a barrier to the diffusion of dissociated and chemisorbed $\mathrm{H}$ to the interior. Alternatively, the catalytic activity of the material may be controversial, as is the case for carbon nanotubes and fullerenes under high pressure [20]. Subject to the requirement for a muon-host interaction mechanism, $\mu \mathrm{SR}$ could be a valuable technique to indicate that substantial $\mathrm{H}$ (as distinct from $\mathrm{H}_{2}$ ) capacity could be available once the surface problems were overcome.

A further example of the potential of $\mu \mathrm{SR}$ for clarification in this context is the role of impurities. While impurities in the carbon structure derived from ball milling [21] or other preparative treatments such as arc discharge [3] are usually viewed as undesirable [6], adventitious metal atoms or clusters may in fact catalyse the dissociation of the hydrogen molecule and facilitate the absorption of hydrogen atoms by carbons. If so, these impurities could be the origin of some of the disparity in measured hydrogen capacities reported in the literature.

The requirement for a muon-host interaction is a drawback for hosts consisting of pure ${ }^{12} \mathrm{C}$, which has essentially zero nuclear dipole moment and no electronic dipole moment. Even here, recent reports of "magnetic carbon" [22] suggest that certain carbon-only structures may be amenable to investigation by $\mu \mathrm{SR}$. Otherwise, there is sufficient interest in and controversy surrounding the absorption of hydrogen by doped carbons [5-7] to provide a rich field of investigation. Broadly speaking, this means dopants with odd mass number, which should possess a nuclear dipole moment. $\mu \mathrm{SR}$ should be especially relevant to Li-doped carbons, as natural $\mathrm{Li}$, which is predominantly ${ }^{7} \mathrm{Li}$, has a much higher nuclear moment than $\mathrm{K}$, in fact.

Conclusions. - Our muon spin relaxation study of pristine graphite and two potassiumintercalated graphites shows no evidence of muon trapping in graphite. In contrast, the appearance of a significant Kubo-Toyabe dip in the $\mathrm{C}_{8} \mathrm{~K}$ and $\mathrm{C}_{24} \mathrm{~K}$ spectra at low temperatures demonstrates positive muon trapping and implies that hydrogen atoms should also be trapped. These results correlate excellently with the accepted relative hydrogen capacities of all three materials and demonstrate the power of $\mu \mathrm{SR}$ for qualitative exploration of the $\mathrm{H}$ storage potential of new materials and clarification of some of the controversial results reported for known materials.

The authors thank J. W. White and G. G. LockhART for the graphite and GIC samples. This work was supported by the Australian Research Council and by the Australian Government's Access to Major Research Facilities Program. We thank J. S. LorD for his assistance in setting up the experiments and F. L. PRATT for providing the WIMDA analysis code. 


\section{REFERENCES}

[1] Dillon A. C. et al., Nature, 386 (1997) 377.

[2] Chambers A., Park C., Baker R. T. K. and Rodriguez N. M., J. Phys. Chem. B., 122 (1998) 4253.

[3] LiU C. et al., Science, 286 (1999) 1127.

[4] Chen P., Wu X., Lin J. and Tan K. L., Science, 285 (1999) 91.

[5] Schlapbach L. and Züttel A., Nature, 414 (2001) 353.

[6] Hirscher M. et al., J. Alloys Compounds, 330-332 (2002) 654.

[7] Gundiah G., Govindaraj A., Rajalakshmi N., Dhatathreyan K. S. and Rao C. N. R., J. Mater. Chem., 13 (2003) 209.

[8] Yang R. T., Carbon, 38 (2000) 623.

[9] Pinkerton F. E. et al., J. Phys. Chem. B, 104 (2000) 9460.

[10] Klyamkin S. N., Nalimova V. A., Semenenko K. N. and Karih A. A., J. Alloys Compounds, 231 (1995) 740.

[11] Chakhalian J. et al., Hyperfine Interact., 106 (1997) 245.

[12] Cox S. F. J. et al., J. Phys. Condens. Matter, 13 (2001) 2169.

[13] Lagrange P., Métrot A. and Hérold A., C.R. Acad. Sci. Paris, 278 (1974) 701.

[14] Watanabe K., Kondow T., Soma M., Onishi T. and Tamaru K., Proc. R. Soc. London, Ser. A, 333 (1973) 51.

[15] Pratt F. L., Physica B, 289-290 (2000) 710.

[16] Hayano R. S., Uemura Y. J., Imazato I., Nishida N., Yamazaki T. and Kybo R., Phys. Rev. B, 20 (1979) 850.

[17] Krimmel H., Schimmele L., Elsässer C. and Fähnle M., J. Phys. Condens. Matter, 6 (1994) 7679.

[18] Terai T. and Takahashi Y., Carbon, 22 (1984) 91.

[19] Enoki T., Sano M. and Inokuchi H., Phys. Rev. B, 32 (1985) 2497.

[20] Chan S. P., Chen G., Gong X. G. and Liu Z. F., Phys. Rev. Lett., 87 (2001) 205502.

[21] Orimo S. et al., Appl. Phys. Lett., 75 (1999) 3093.

[22] Makarova T. L. et al., Nature, 413 (2001) 716. 